\title{
Scientific publications in urology and nephrology journals from China: A 10-year analysis
}

\author{
Xiaomei Zhou, MD; ; Changyong Xing, MD; ${ }^{*}$ Lei Xin, MD; Hongzhen Hu, MD; ${ }^{*}$ Liping Li, MD; ${ }^{*}$ Jingchuan Fang, \\ $M D^{*}$ Zhiyong Liu, MD
}

*Shenzhen Futian Maternity \& Infant Health Hospital, Shenzhen, China; 'Department of Urology, Shanghai Changhai Hospital, Second Military Medical University, Shanghai, China

Cite as: Can Urol Assoc J 2012;6(2):102-6. http://dx.doi.org/10.5489/cuaj.11125

\begin{abstract}
Background: The scientific research in urology and nephrology of China has developed significantly. The present study was designed to analyze the outputs of publications in urology and nephrology journals from three regions of China: mainland, Taiwan and Hong Kong.

Methods: The numbers of articles, impact factors, citation reports and other indexes within this category between 2000 and 2009 were extracted for quantity and quality comparisons from PubMed and the ISI (Institute for Scientific Information-currently called the Thomson Reuters Web of Knowledge) database.

Results: There were 3100 articles from the mainland $(36.5 \%)$, Taiwan (46.8\%) and Hong Kong (16.7\%), and the increasing trend in each region was significant $(p<0.001)$. The accumulated impact factor and total citation of Taiwan exceeded the other two regions, while the average impact factor and citation of Hong Kong was highest. There were differences between the three regions on the most popular journals.

Interpretation: Although the quantity of articles in urology and nephrology from the mainland has exceeded Taiwan and Hong Kong since 2008, there is a considerable gap in the quality of articles between the mainland and the other two regions.
\end{abstract}

\section{Introduction}

Over the past several decades, the overall health situation of Chinese people, especially in the mainland, has improved gradually. However, the prevalence of urinary and kidney disease has been increasing rapidly in China in recent years. These diseases have become a major public health problem for the country. For example, the incidence of prostate cancer, which was very low in China, has now become the most prevalent urogenital cancer in men with an overall incidence rate of $4.3 / 10^{5}$ person year in $2008 .^{1}$ Correspondingly, the research (basic or clinical) in urology and nephrology is developing dramatically. Since the quantity and quality of research articles published in scientific journals can reflect the research productivity of a country, ${ }^{2}$ we analyzed the contribution of scientific publications in urology and nephrology from Chinese authors in three major regions of China (the mainland $[\mathrm{ML}]$, Hong Kong $[\mathrm{HK}]$ and Taiwan $[\mathrm{TW}]$ ).

\section{Methods}

A total of 63 journals were included in the "Urology \& Nephrology" category of Science Citation Index Expanded (SCIE) subject categories by the Institute for Scientific Information (ISI). We excluded journals which were not indexed by PubMed; in the final analysis, we included 59 journals. A computerized literature search was conducted in the PubMed database on October 1, 2010, followed by manual classification according to the exact publication date of each paper. Articles published from ML, TW, and HK between 2000 and 2009 in these journals were elicited. The International Standard Serial Number (ISSN-for print) was used to perform searches in PubMed. Taiwan [ad]", "Hong Kong [ad]" and "China [ad] NOT Taiwan [ad] NOT Hong Kong [ad]" were the keywords for addresses. Articles that showed the first author's affiliation with the three regions were considered as research output from the regions. The number of clinical trials, randomized controlled trials (RCT) and case reports were generated according to the publication types by PubMed.

Web of Science was used for quality comparison and inclusion/exclusion criteria were delineated before the start of the literature search. To reflect the real research situation and avoid repeat inclusion, only original articles and reviews were extracted and other publication types such as editorial, meeting abstract and letter were excluded. Moreover, since ISI cannot search articles by limiting first author's address, a manual check was performed to weed out different countries and regions. Articles meeting the inclusion/exclusion criteria above were first extracted independently and collaboratively 
if there was a disagreement between the reviewers (Zhou X and Xing C). Three methods were used to compare the quality of research articles in these three regions. First, the accumulated impact factor (IF) and the average IF were generated according to the journal citation reports (JCR) 2009. Second, the citation reports of articles affiliated with a Chinese institution were conducted. Third, articles published in the top 10 high-impact journals in urology and nephrology were generated and analyzed. Furthermore, we determined the top 10 popular urology and nephrology journals in these three regions according to the number of published articles.

Statistical analyses were performed using SPSS 13.0 (SPSS, Chicago, IL). The nonparametric test for trend was performed to determine any significant change of the total numbers over the period of time. The Kruskal-Wallis test was used to detect the difference among the three regions, and the rank-sum test was used to detect the difference between two regions when necessary. The test for significance was two-tailed and a $p$ value $<0.05$ was considered significant.

\section{Results}

\section{Total number of articles and trends}

In total, 98215 articles were published in the selected 59 urology and nephrology journals from 2000 to 2009 worldwide. There were 3100 articles from three regions of China: ML (1131/3100, 36.5\%), TW (1452/3100, 46.8\%) and HK $(517 / 3100,16.7 \%)$. There was a significant increase from 2000 to 2009 in the three regions: ML from 29 to 303, TW from 77 to 220 and in HK from 33 to $51(p<0.001)$ (Fig. 1). From 2008 onwards, the number of articles published from ML exceeded those from TW or HK, and even exceeded the combined number of articles published from TW and HK in 2009. The share of articles published in the

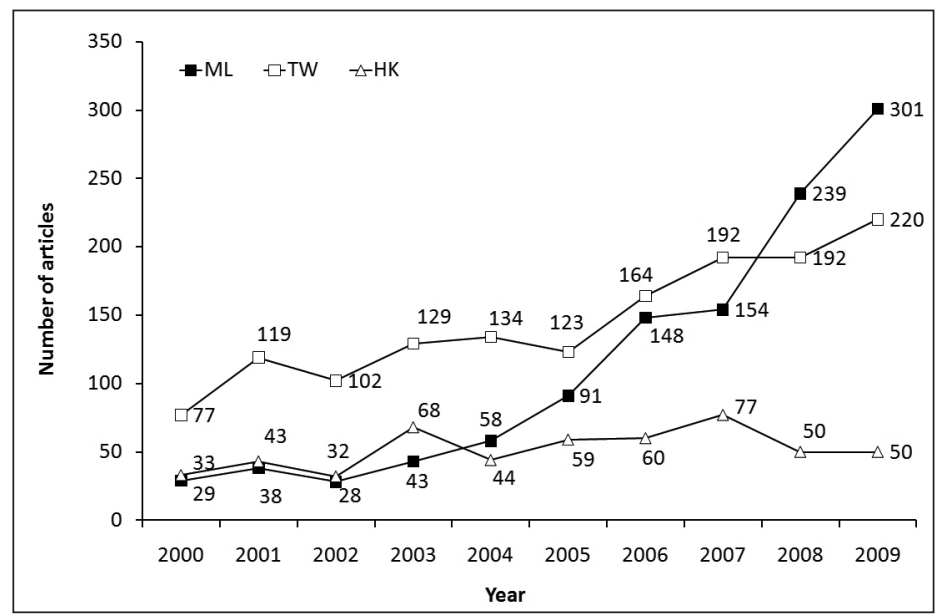

Fig. 1. Number of articles from mainland, Taiwan and Hong Kong in urology and nephrology between 2000 and 2009.
ML and TW compared with the total number of articles published in urology and nephrology increased significantly ( $p<0.001$, for both regions); HK showed no statistical difference $(p=0.233)$.

\section{Clinical trials, RCT, and case report}

Regarding the number of clinical trials published, there was a significant difference among the three regions $(M L=95$; $\mathrm{TW}=157 ; \mathrm{HK}=54, p<0.001)$. A similar difference was found for RCTs $(\mathrm{ML}=50$; TW $=69 ; \mathrm{HK}=36, p<0.001)$ and case reports $(\mathrm{ML}=64 ; \mathrm{TW}=373 ; \mathrm{HK}=72, p<0.001)$ (Fig. 2). In these three comparisons, the number of articles from TW exceeded the other two regions (both $p<0.001$ ).

\section{Impact factor}

The impact factor is a measure reflecting the average number of citations to recent articles published in science and social science journals. According to the JCR 2009, Nature Reviews Nephrology and Nature Reviews Urology had no IF. After excluding these journals and the 107 articles no first authors from China, 3002 articles or reviews were retrieved from Web of Science $(M L=1194 ; T W=1368 ; H K=440)$. Accumulated IFs of ML, TW and HK were 3210.612, 3725.080 and 1524.173 with significant difference $(p=0.008)$ during the past 10 years. Accumulated IF of TW was higher than HK, but comparable with ML (TW vs. HK $p<0.001$; TW vs. ML $p=0.481$ ). It still seemed that the average IF of articles from three regions was different significantly (ML: 2.689, TW: 2.723, HK: $3.464, p<0.001)$. The average IF of HK was higher than ML and TW (both $p=0.001$ ), while there was no difference between ML and TW ( $p=0.821)$ (Table 1).

\section{Citation reports}

In this analysis, TW got the highest number of total citations (9981), followed by ML (7059) and HK (5525) ( $p=0.054)$. However, if this comparison was limited to 2005-2009, ML got the highest number of total citations $(p=0.061)$. Regarding the average citation for each article, HK got the highest citation (12.56), followed by TW (7.30) and $M L$ (5.91), but this trend was not statistically significant $(p=0.153)$ (Table 2$)$.

\section{High-impact journals}

A total number of 805 articles from the three regions were published in the 10 top-ranking urology and nephrology journals. In total, nearly two thirds (522/805, 64.8\%) were clustered in three journals: Journal of Urology, Kidney International and American Journal of Kidney Diseases. Of the papers from TW, 361 were published in the 10 top- 
Zhou et al.

\begin{tabular}{|c|c|c|c|c|c|c|}
\hline \multirow{2}{*}{ Year } & \multicolumn{3}{|c|}{ Accumulated IF } & \multicolumn{3}{|c|}{ Average IF } \\
\hline & ML & TW & HK & ML & TW & HK \\
\hline 2000 & 54.841 & 263.996 & 119.349 & 1.891 & 3.219 & 3.510 \\
\hline 2001 & 89.108 & 362.328 & 184.865 & 2.345 & 3.178 & 4.108 \\
\hline 2002 & 85.588 & 293.298 & 148.338 & 2.951 & 2.716 & 4.495 \\
\hline 2003 & 156.152 & 353.086 & 170.921 & 3.123 & 2.675 & 3.637 \\
\hline 2004 & 162.236 & 330.029 & 137.545 & 2.750 & 2.558 & 3.355 \\
\hline 2005 & 322.290 & 368.288 & 153.064 & 3.040 & 2.855 & 2.944 \\
\hline 2006 & 472.338 & 439.881 & 163.050 & 2.795 & 2.767 & 3.469 \\
\hline 2007 & 447.600 & 416.549 & 173.935 & 2.713 & 2.620 & 3.162 \\
\hline 2008 & 662.147 & 413.725 & 126.581 & 2.638 & 2.492 & 3.014 \\
\hline 2009 & 758.312 & 483.900 & 146.525 & 2.536 & 2.547 & 3.256 \\
\hline Total & 3210.612 & 3725.080 & 1524.173 & 2.689 & 2.723 & 3.464 \\
\hline
\end{tabular}

IF: impact factor; ML: mainland; TW: Taiwan; HK: Hong Kong.

ranking journals, compared with 276 and 168 articles from $\mathrm{ML}$ and $\mathrm{HK}$, respectively (Table 3 ).

\section{Popular journals}

The Asian Journal of Andrology was the most popular journal in ML, while Urology and American Journal of Kidney Diseases was most popular in TW and HK, respectively (Table 4). Five journals (Nephrology, Kidney International, Nephrology Dialysis Transplantation, BJU International and Journal of Urology) were among the top 10 nephrology and urology journals in all the three regions (Table 4).

\section{Discussion}

In the present study, we analyzed the quantity and quality of articles in urology and nephrology from three major regions of China to reflect the situation of scientific research in these fields. The results showed a significant increase in the number of articles published in these three regions (especially in

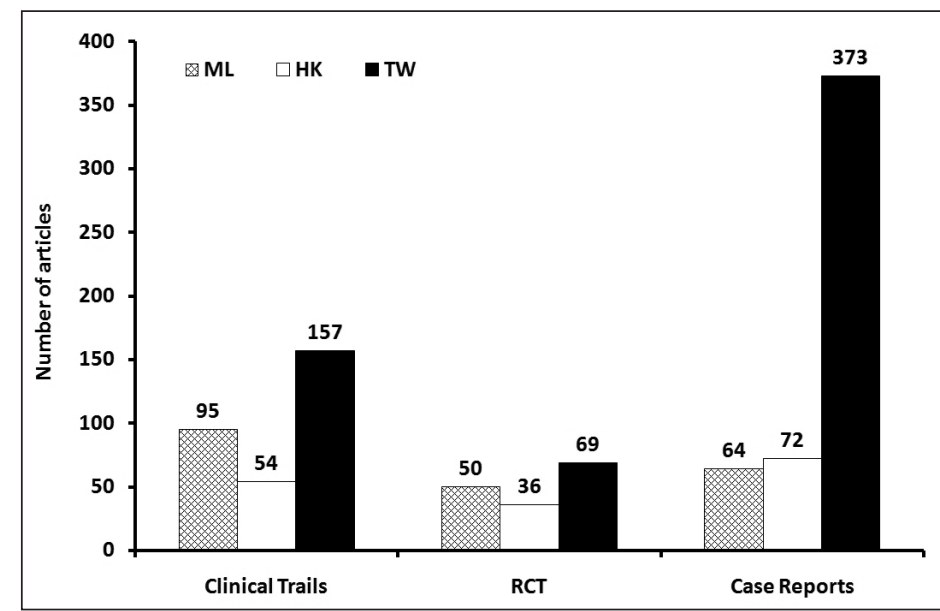

Fig. 2. Number of articles on clinical trials, randomized controlled trials, and case reports from the mainland, Taiwan and Hong Kong. the $\mathrm{ML}$ ) in the past decade. However, if this comparison represented the quality of the articles, the accumulated number from TW exceeded the other two regions, while the average number of published articles was higher in HK.

Publishing in scientific journals is the best way to introduce new medical information and clinical applications to a large audience of physicians. ${ }^{3}$ The quality and quantity of articles determines how new information is incorporated into the current standard of care and leads the way to future advances. It also is a way to evaluate the scientific productivity of an institution or country. Since urology and nephrology research is advancing dramatically, the assessment of scientific publication can reflect the level of science and research of some regions. ${ }^{4}$

The ML of China has achieved notable advancements in scientific research during these decades, especially in urology and nephrology. The number of articles published by Chinese authors in international urology and nephrology journals has increased significantly. As this study demonstrated, the number of publications significantly increased from 29 to 303 during the past 10 years and the total citation of articles from ML has exceeded TW and HK between 2005 and 2009. There are four reasons for this increase. Firstly, the ML has a huge population (ML: 1341 million in 2010, TW: 23 million in 2011, HK: 7.1 million in $2010^{5-7}$ ), supplying subjects for investigation. Secondly, funding in the field of life sciences and medicine, including urology and nephrology research, has increased considerably. The ML spent $2.21 \%$ of its gross domestic product on research and development in $2007 ;{ }^{8}$ this number is expected to increase to $2.5 \%$ by 2020. ${ }^{9}$ Thirdly, in recent years, more and more "brain drain" of China has returned home. Because of their experience and capabilities brought from host countries, Chinese scientists are on the frontier of research and produce high-quality articles. ${ }^{10}$ Finally, there is a publish-or-perish mentality in China, with a focus on Western journals. In some top-tier research institutions in China, Science Citation Index (SCl) journals 
Publications in urology and nephrology from China

\begin{tabular}{|c|c|c|c|c|c|c|}
\hline \multirow{2}{*}{ Year } & \multicolumn{3}{|c|}{ Total citation } & \multicolumn{3}{|c|}{ Average citation } \\
\hline & ML & TW & HK & ML & TW & HK \\
\hline 2000 & 195 & 1037 & 843 & 6.72 & 12.65 & 24.79 \\
\hline 2001 & 290 & 1403 & 845 & 7.63 & 12.31 & 18.78 \\
\hline 2002 & 294 & 1216 & 717 & 10.14 & 11.26 & 21.73 \\
\hline 2003 & 741 & 1418 & 777 & 14.82 & 10.74 & 16.53 \\
\hline 2004 & 645 & 1302 & 460 & 10.93 & 10.09 & 11.22 \\
\hline 2005 & 1213 & 1077 & 632 & 11.55 & 8.35 & 12.15 \\
\hline 2006 & 1427 & 1174 & 604 & 8.44 & 7.38 & 12.85 \\
\hline 2007 & 917 & 680 & 377 & 5.56 & 4.28 & 6.85 \\
\hline 2008 & 940 & 444 & 175 & 3.75 & 2.67 & 4.17 \\
\hline 2009 & 397 & 230 & 95 & 1.33 & 1.21 & 2.16 \\
\hline Total & 7059 & 9981 & 5525 & 5.91 & 7.30 & 12.56 \\
\hline
\end{tabular}

have become the required outlet for research. ${ }^{11}$ The $\mathrm{SCl}$ is also a part of the performance review process in China; there are many approvals and rewards in scientific research in the ML. This phenomenon may explain the relatively high quantity and low quality of articles from the ML and why this region does not lead in IF and number of citations compared with TW and HK.

Clinical trials, especially RCTs, are the gold standard for evidence-based assessment of therapeutic interventions. It is believed that there are several advantages for clinical trials in the ML, due to its large-scale subjects, good compliance and relatively low cost. ${ }^{12}$ However, our results did not find an advantage in clinical trials published from the ML. There were 50, 69 and 36 RCTs reported from the ML, TW and HK, respectively. If population base is considered, there was a large gap between the ML and the other two regions. Considering that industry sponsorship plays an important role in clinical research, ${ }^{13,14}$ a possible reason is the different degree of industry involvement in this type of research between these regions. Recently, more and more pharmaceutical companies focus on the $\mathrm{ML}$, so we expect the number of clinical studies from this region to increase.

Investigations from HK and TW have contributed some of the best scientific papers from the three regions, especially when IF, citation reports and articles published in top general medicine journals were compared. ${ }^{15,16} \mathrm{IF}$ is highly associated with the quality of a journal, but not an article. For example, when we consider the average IF of each article, HK preceded TW and ML (3.464 vs. 2.723 vs. 2.689, respectively, $p<0.001$ ); this implies that research results from HK in urology and nephrology are more acceptable to highimpact journals. HK also got the highest citation (12.56), followed by TW (7.30) and the ML (5.91). Comparing IF and citations of three regions, we found that articles from HK in urology and nephrology appeared to be of highest quality.

There are some limitations in our study. First, citations and IF are used for quality comparison, both of which are easy and objective parameters, but are not perfect indicators. The prominence of a journal, measured by its IF, influences the number of citations, ${ }^{17}$ so a circular phenomenon

\begin{tabular}{|c|c|c|c|c|c|c|c|c|c|c|}
\hline Rank & Journal & 2009 IF & TW & $\%$ & ML & $\%$ & HK & $\%$ & Total & $\%$ \\
\hline 1 & J Am Soc Nephrol & 7.689 & 29 & $0.00 \%$ & 44 & $50.00 \%$ & 30 & $50.00 \%$ & 103 & $12.80 \%$ \\
\hline 2 & Eur Urol & 7.667 & 18 & $58.06 \%$ & 11 & $35.48 \%$ & 2 & $6.45 \%$ & 31 & $3.85 \%$ \\
\hline 3 & Kidney Int & 6.193 & 51 & $30.54 \%$ & 79 & $47.31 \%$ & 37 & $22.16 \%$ & 167 & $20.75 \%$ \\
\hline 4 & Am J Kidney Dis & 5.152 & 86 & $53.75 \%$ & 13 & $8.13 \%$ & 61 & $38.13 \%$ & 160 & $19.88 \%$ \\
\hline 5 & Nat Clin Pract Nephr & 4.938 & 0 & $0.00 \%$ & 1 & $50.00 \%$ & 1 & $50.00 \%$ & 2 & $0.25 \%$ \\
\hline 6 & J Sex Med & 4.884 & 17 & $58.62 \%$ & 8 & $27.59 \%$ & 4 & $13.79 \%$ & 29 & $3.60 \%$ \\
\hline 7 & Clin J Am Soc Nephro & 4.844 & 4 & $26.67 \%$ & 3 & $20.00 \%$ & 8 & $53.33 \%$ & 15 & $1.86 \%$ \\
\hline 8 & J Urology & 4.016 & 120 & $61.54 \%$ & 62 & $31.79 \%$ & 13 & $6.67 \%$ & 195 & $24.22 \%$ \\
\hline 9 & Curr Opin Nephrol Hy & 3.961 & 2 & $40.00 \%$ & 2 & $40.00 \%$ & 1 & $20.00 \%$ & 5 & $0.62 \%$ \\
\hline \multirow[t]{2}{*}{10} & Am J Nephrol & 3.481 & 34 & $34.69 \%$ & 53 & $54.08 \%$ & 11 & $11.22 \%$ & 98 & $12.17 \%$ \\
\hline & Total & & 361 & $44.84 \%$ & 276 & $34.29 \%$ & 168 & $20.87 \%$ & 805 & $100.00 \%$ \\
\hline
\end{tabular}

IF: impact factor; ML: mainland; TW: Taiwan; HK: Hong Kong; J Am Soc Nephrol: Journal of the American Society of Nephrology; Eur Urol: European Urology; Kidney Int: Kidney International; Am J Kidney Dis: American Journal of Kidney Diseases; Nat Clin Pract Nephr: Nature Clinical Practice Nephrology; J Sex Med: The Journal of Sexual Medicine; Clin J Am Soc Nephro: Clinical Journal of the American Society of Nephrology; J Urology: Journal of Urology; Curr Opin Nephrol Hy: Current Opinion in Nephrology and Hypertension; Am J Nephrol: American Journal of Nephrology. 


\begin{tabular}{|c|c|c|c|c|c|c|}
\hline Rank & ML & $\mathbf{n}$ & TW & $\mathbf{n}$ & HK & $\mathbf{n}$ \\
\hline 1 & Asian J Androl (IF: 1.688) & 229 & Urology (IF: 2.365) & 155 & Am J Kidney Dis (IF: 5.152) & 61 \\
\hline 2 & Urology (IF: 2.365) & 82 & Renal Failure (IF: 0.84) & 126 & Nephrol Dial Transpl (IF: 3.306) & 54 \\
\hline 3 & Kidney Int (IF: 6.193) & 79 & J Urology (IF: 4.016) & 120 & Periton Dialysis Int (IF: 1.636 & 43 \\
\hline 4 & Nephrol Dial Transpl (IF: 3.306) & 65 & Nephrol Dial Transpl (IF: 3.306) & 118 & Kidney Int (IF: 6.193) & 37 \\
\hline 5 & J Urology (IF: 4.016) & 62 & Am J Kidney Dis (IF: 5.152) & 86 & J Am Soc Nephrol (IF: 7.689) & 29 \\
\hline 6 & BJU Int (IF: 2.865) & 53 & Urol Int (IF: 0.902) & 68 & Nephrology (IF: 1.219) & 28 \\
\hline 7 & Nephrology (IF: 1.219) & 52 & Clin Nephrol (IF: 1.373) & 64 & Asian J Androl (IF: 1.688) & 16 \\
\hline 8 & Am J Nephrol (IF: 3.481) & 51 & BJU Int (IF: 2.865) & 59 & BJU Int (IF: 2.865) & 14 \\
\hline 9 & Clin J Am Soc Nephrol (IF: 7.689) & 44 & Kidney Int (IF: 6.193) & 51 & J Urology (IF: 4.016) & 13 \\
\hline 10 & Urol Int (IF: 0.902) & 39 & Nephrology (IF: 1.219) & 38 & Prostate (IF: 3.081) & 12 \\
\hline
\end{tabular}

IF: impact factor; ML: mainland; TW: Taiwan; HK: Hong Kong; Am J Kidney Dis: American Journal of Kidney Diseases; Am J Nephrol: American Journal of Nephrology; Kidney Int: Kidney International; Clin J Am Soc Nephro: Clinical Journal of the American Society of Nephrology; J Urology: Journal of Urology; J Am Soc Nephrol: Journal of the American Society of Nephrology; Asian J Androl: Asian Journal of Andrology; BJU Int: British Journal of Urology International; Clin Nephrol: Clinical Nephrology; J Am Soc Nephrol: Journal of the American Society of Nephrology; Nephrol Dial Transpl: Nephrology Dialysis Transplantation. Periton Dialysis Int: Peritoneal Dialysis International; Prostate: The Prostate; Urol Int: International Journal of Urology.

develops (good articles get published in prestigious journals with a high IF, which results in more citations and further increases the IF, and so on). Although we supplied data on the total and average IF and citation, the real quality difference may be exaggerated. Second, a proportion of articles were completed by Chinese researchers abroad with the Chinese author as the first author and a foreign author as the corresponding author. However, Pubmed does not supply the corresponding author's affiliation, so we could not exclude these publications by computerized filter; this may have led to a confounding bias.

\section{Conclusion}

The quantity of scientific articles from three regions of China has increased significantly in the past decade. However, there are considerable gaps between the quality of articles among the ML, TW and HK, particularly when in average IF and citations. Authors from the ML need further focus on the quality of their research articles beyond the quantity of publications.

Competing interests: None declared.

This paper has been peer-reviewed.

\section{References}

1. Zhang L, Yang BX, Zhang HT, et al. Prostate cancer: an emerging threat to the health of aging men in Asia. Asian J Androl 201 1;13:574-8. http://dx.doi.org/10.1038/aja.2010.126. Epub 2011 May 9.

2. Cappell MS, Davis M. A significant decline in the American domination of research in gastroenterology with increasing globalization from 1980 to 2005: An analysis of American authorship among 8,251 articles. Am J Gastroenterol 2008;103:1065-74.http://dx.doi.org/10.1111/i.1572-0241.2007.01767.x
3. Sorrentino D, De Biase F, Trevisi A, et al. Scientific publications in gastroenterology and hepatology in Western Europe, USA and Japan in the years 1992-1996: A global survey. Digestion 2000;61:77-83. http://dx.doi.org/10.1159/000007738

4. Scales CD Jr, Norris RD, Keitz SA, et al. A critical assessment of the quality of reporting of randomized, controlled trials in the urology literature. J Urol 2007;177:1090-4.http://dx.doi.org/10.1016/i. juro.2006.10.027

5. National Economic and Social Development Statistical Communique of People's Republic of China 2010. http://www.stats.gov.cn/tigb/ndtigb/qgndtigb/t20110228_402705692.htm [in Chinese]. Accessed March 6, 2012.

6. Monthly Bulletin of Interior Statistics 2011. http://sowf.moi.gov.tw/stat/month/elist.htm. Accessed March 6, 2012

7. Population and Vital Events. httrp://www.censtatd.gov.hk/hong_kong_statistics/statistics_by_subject/ index.jp? subjectID=1\&charset|D=2\&displayMode=T. Accessed March 6, 2012.

8. Research and development expenditure (\% of GDP) of China in 2007. http://data.worldbank.org/topic/ science-and-technology. Accessed March 6, 2012.

9. Wells WA. The returning tide: How China, the world's most populous country, is building a competitive research base. J Cell Biol 2007;176:376-401. http://dx.doi.org/10.1083/ich.200701150

10. Jonkers K, Tijssen R. Chinese researchers returning home: Impacts of international mobility on research collaboration and scientific productivity. Scientometrics 2008;77:309-33. http://dx.doi.org/10.1007/ s11192-007-1971-x

11. Xue L. China: The prizes and pitfalls of progress. Nature 2008;454:398-401.http://dx.doi. org $/ 10.1038 / 454398$ a

12. Mervis J. Epidemiology. China's unique environment favors large intervention trials. Science 1995;270:1149-51. http://dx.doi.org/10.1126/science.270.5239.1149

13. Montaner JS, O'Shaughnessy MV, Schechter MT. Industry-sponsored clinical research: a double-edged sword. Lancet 2001;358:1893-5. http://dx.doi.org/10.1016/S0140-6736(01)06891-X

14. Angell M. Industry-sponsored clinical research: a broken system. JAMA 2008;300:1069-71. http:// dx.doi.org/10.1001/jama.300.9.1069

15. Hu LH, Liao Z, Gao R, et al. Scientific publications in cardiology and cardiovasology journals from Chinese authors in various parts of north Asia: 10-year survey of literature. Int J Cardiol 2010; 140:304-8. http:// dx.doi.org/10.1016/i.i.icard.2008.11.086

16. Li G, Hu LH, Liao Z, et al. Scientific publications in pharmacology and pharmacy jo urnals from Chinese authors in various parts of North Asia: a 10-year survey of the literature. J Int Med Res 2010;38:750-9.

17. Perneger TV. Citation analysis of identical consensus statements revealed journal-related bias. I Clin Epidemiol 2010;63:660-4. htrp://dx.doi.org/10.1016/i.jlinepi.2009.09.012

Correspondence: Dr. Zhiyong Liu, Department of Urology, Shanghai Changhai Hospital, Second Military Medical University, 168 Changhai Rd, Shanghai 200433, China; fax: +86-21-55620081; urology_liu@163.com 